\title{
Induction of Immune Response in Animal Model Using Recombinant Anti-NDV Vaccine
}

\author{
Amir Ghaffar Shahriari ${ }^{1,}$, Abdolreza Bagheri ${ }^{2}$, Alireza Afsharifar ${ }^{3}$, Maziar Habibi-Pirkoohi ${ }^{4}$ \\ ${ }^{1}$ Department of Agriculture and Natural Resources, Higher Education Center of Eghlid, Eghlid, Iran \\ ${ }^{2}$ Department of Crop Biotechnology and Breeding, Ferdowsi University of Mashhad, Mashhad, Iran \\ ${ }^{3}$ Plant Virology Research Centre, College of Agriculture, Shiraz University, Shiraz, Iran \\ ${ }^{4}$ Research and Technology Institute of Plant Production, Shahid Bahonar University of Kerman, Iran \\ * Corresponding author: Amir Ghaffar Shahriari, Higher Education Center of Eghlid, Eghlid, Iran. Tel/Fax: +98-7144534056; E-mail: \\ Shahriari.ag@eghlid.ac.ir
}

\begin{abstract}
Background: Newcastle disease is a major avian disease that causes enormous economic loss in poultry industry. There have been a number of reports on the suitability of plant-based recombinant vaccine against this disease. Fusion (F) and hemagglutinin-neuraminidase (HN) epitopes of the Newcastle disease virus (NDV) represent the major immunogenic sites for development of recombinant anti-ND vaccines in plant hosts.

Objectives: The main objective of this research was to evaluate the ability of a recombinant anti-ND vaccine in induction of immune responses in animal model.

Materials and Methods: In this study, immunogenicity of recombinant fusion (F) and hemagglutinin-neuraminidase (HN) epitopes of the Newcastle disease virus (NDV) is investigated in an animal model. The corresponding genes encoding amino acids 65-81 of the F protein and 346-353 amino acids of $\mathrm{HN}$ were expressed in tobacco seedling using agrobacterium-mediated transformation. Expression of the foreign gene in the tobacco seedlings was investigated by a number of molecular assays including Real-Time PCR and ELISA. Transgenic plant extract was used to induce immunogenic response in animal model.

Results: Integration of the foreign gene in plant host genome was confirmed by polymerase chain reaction (PCR). Expression of the foreign recombinant protein was confirmed by Real-Time PCR and ELISA assays. Immunogenicity of the recombinant protein was investigated in rabbit by subcutaneous injection. Results indicated that the transgenic plant extract can induce immune responses in the host as confirmed by presence of specific antibodies in the sera in ELISA assay. Western blot assays showed that the foreign gene was actually expressed in transgenic seedlings.

Conclusions: The results obtained in this research provide further evidence on applicability of plant-based recombinant vaccines for protection of poultry against Newcastle disease.
\end{abstract}

Keywords: ELISA; Immunogenic Response; Mucosal Immunity; Newcastle Disease; Recombinant Vaccine; Western Blot

\section{Background}

Due to the progress in genetic engineering, green plants have emerged as ideal platforms for production of recombinant vaccine during recent decades. Various antigens relating to a large number of animal and human diseases have been studied in different plant species for the purpose of producing recombinant vaccines. Despite the unique advantages of plant systems as green factories for production of recombinant vaccines, there are some major hurdles that have prevented commercial production of plant-based vaccines (1). One of the major problems with recombinant vaccines is the inefficacy of the expressed antigens for induction of immune response in animal hosts (2). Oral delivery of plant-based recombinant vaccines is a potential alternative to injection, owing to the low cost and ease of administration. Plant-based vaccines are free of human and animal diseases and, therefore, can reduce the costs of screening for viral and microbial contamination. In addition, the chance of acquiring mucosal immunity against infectious agents that enter the body across a mucosal surface is increased with oral vaccines (3). Despite numerous advantages of green plants over other heterologous expression systems such as bacteria, yeast and insect cell culture, low expression level of the antigen in plant hosts is still a main challenge for commercialization of plant-based recombinant 
vaccines (4). To overcome this problem, various strategies such as host-specific codon optimization, inclusion of 5'-untranslated-leader sequences, insertion of introns within coding region, and the application of signal peptide have been proposed and applied in genetic transformation experiments with varying degrees of success (1). The main problem in the application of orally-delivered immunogenic proteins is that some proteins are degraded after ingestion and some immunogens may not be recognized efficiently at mucosal-immune-effector sites in the gut. Plant-derived vaccines have demonstrated the ability to induce both systemic and mucosal immune responses (5).

Newcastle disease (ND) has been a major target for development of veterinary vaccines during recent decades and many attempts have been made to develop efficient vaccines against ND via genetic engineering in higher plants (6). In our previous work, we successfully transferred and expressed HemagglutininNeuraminidase (HN) and Fusion (F) epitopes of Newcastle Disease Virus (NDV) in tobacco seedlings (7). Herein, we report induction of immune response in animal model using recombinant $\mathrm{HN}$ and $\mathrm{F}$ epitopes as potential recombinant vaccine against ND.

\section{Objectives}

In the present investigation, we report subcutaneous administration of a fusion HN-F peptide and subsequent immune response induction in animal model. The fusion peptide includes four tandem repeat of HN epitope (encoding amino acids 346-353) with 96bp length followed by three tandem repeat of $\mathrm{F}$ epitope (encoding amino acids 65-81) with 153bp length.

\section{Materials and Methods}

\subsection{Plant Transformation}

Gene construct design, Construction of Binary Vector and plant transformation, and verification of putative transgenic lines have been described elsewhere (7).

\subsection{Selection of Transgenic Lines with the Highest} Expression

Real-time PCR and ELISA assays were performed to screen the putative transgenic lines to select those with the highest expression.

\subsubsection{Real-Time PCR}

Expression of the synthetic gene was evaluated in transcription level by real-time PCR assay. Total RNA was extracted from $500 \mathrm{mg}$ of leaf tissue using RNX-Plus according to manufacturers' instruction. Complementary DNA (cDNA) was synthesized via reverse transcription using oligo $(\mathrm{dT})_{20}$ primer. The resulting cDNA mixtures were used as templates for the real-time PCR. Expression of the synthetic gene was quantitatively analyzed using a real-time PCR system
(BioRad). Real-time PCR was carried out in a $20 \mu \mathrm{L}$ reaction volume containing $0.5 \mu \mathrm{M}$ of each primer and $10 \mu \mathrm{L}$ of SYBR Green real-time PCR master mix (Genet Bio, South Korea). Quantitative real-time PCR experiments were performed in duplicate for each sample. Forward and reverse primers for real-time PCR were 5'-GCCACACCTCGCACATTG $3{ }^{\prime}$ and $5{ }^{\prime}$ GCCAGCATCACCATTCTTG-3', respectively.

\subsubsection{ELISA}

ELISA plate was coated with total soluble proteins from the wild type and the transformed plants and purified NDV particles (commercial vaccine) at $37^{\circ} \mathrm{C}$ for one hour, followed by incubation with $1 \%$ bovine serum albumin (BSA) in PBS for $2 \mathrm{~h}$ at $37^{\circ} \mathrm{C}$ to prevent nonspecific binding. The well was washed by PBST/PBS and incubated with anti-NDV serum. Afterwards, it was incubated with horseradish peroxidase conjugated with anti-rabbit IgG (1:1500). The anti-NDV serum was prepared after three times of immunization in rabbit with the inactivated V4 NDV particles and diluted at 1:1000 in PBST. Wells were developed with TMB substrate. The color reaction was stopped by $2 \mathrm{~N}$ $\mathrm{H} 2 \mathrm{SO} 4$ and read at $450 \mathrm{~nm}$ of wavelength.

\subsection{Western Blot Assay}

Total soluble protein (TSP) was extracted from transgenic tobacco plants as described by (4). Briefly, $1 \mathrm{gr}$ of fresh leaves was ground in liquid nitrogen. One milliliter of extraction buffer was added, and the homogenate was centrifuged at $13,000 \times \mathrm{g}$ for $15 \mathrm{~min}$ at $4{ }^{\circ} \mathrm{C}$ and the supernatant was used for further analysis. Protein samples were resolved by $15 \%$ SDSpolyacrylamide gel electrophoresis and transferred onto PVDF at $130 \mathrm{~mA}$ for $2 \mathrm{~h}$. To prevent nonspecific antibody reactions, the membrane was blocked with BSA. After blocking with BSA, the membrane was washed using PBST/PBS, and incubated with horseradish peroxidase conjugated with anti his tag. After washing three times, the reaction was developed by addition of the TMB.

\subsection{Induction of Immune Response in Rabbit}

Rabbits were immunized on days 0, 15, 29 and 50 with $500 \mu \mathrm{g}$ of crude protein of wild type and transgenic plant (T26) emulsified in Incomplete Freund's adjuvant. Animals were then bled and the presence of anti-NDV specific antibodies in sera was determined by ELISA, in which the plates were coated with purified NDV.

\section{Results}

\subsection{Real-Time PCR}

The results of real-time PCR are presented in Figure 1. As seen, the highest level of transgene transcription can be observed in lines T3, T12 and T26. This finding implies that lines T26, T12 had the highest expression level compared to others. 


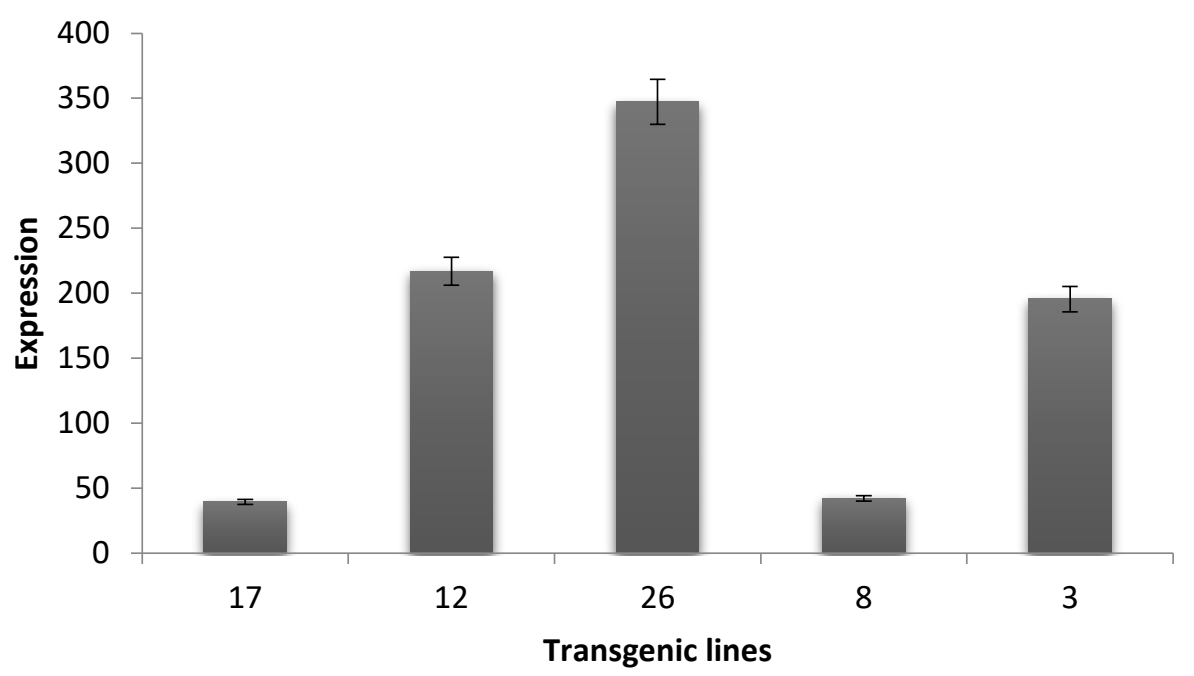

Figure 1. The results of real-time PCR

\subsection{ELISA Assay}

The results of ELISA assay are presented in Figure 2. As seen, the highest expression level was observed in lines $\mathrm{T} 26, \mathrm{~T} 12$ and $\mathrm{T} 3$.

The lines with higher expression of the recombinant protein were used for further analyses. The assay was conducted in two replications.

\subsection{Western-Blot Assay}

Expression of $\mathrm{HN}-\mathrm{F}$ fusion peptide in the transgenic lines was evaluated by the western-blot assay (Fig. 3). As can be seen in Figure 3, an about $10.6 \mathrm{kDa}$ protein band appeared obviously in the transformed lines T26 and $\mathrm{T} 12$ whereas wild type and other transgenic lines showed no band.
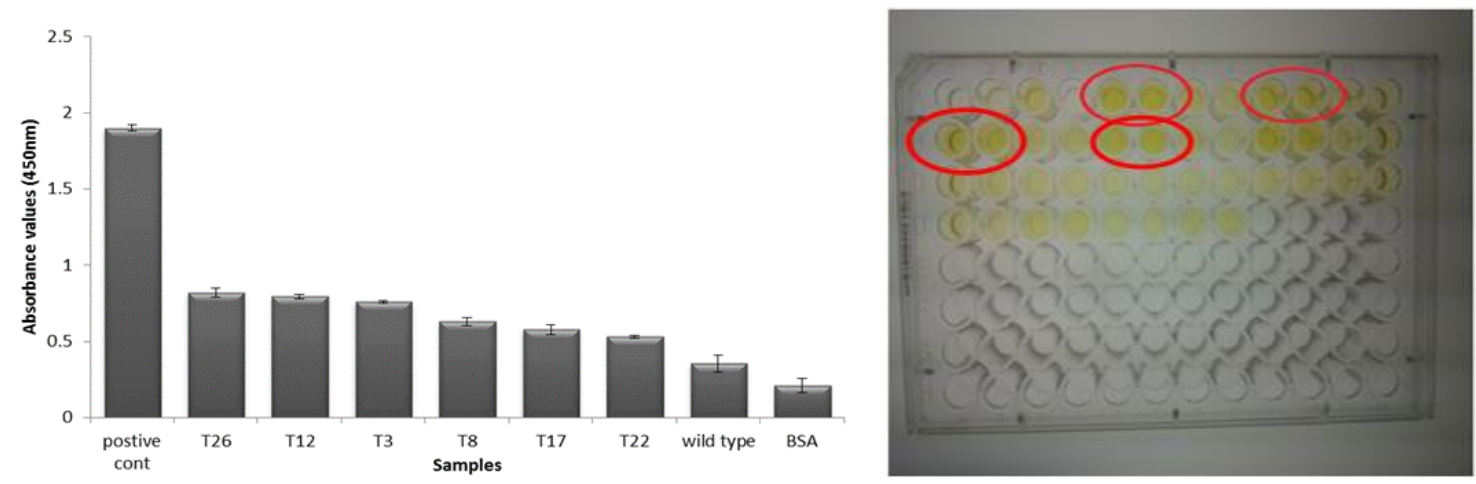

Figure 2. ELISA analysis of transgenic plants. The wells surrounded by red circles indicates positive control, T3, T12 and T26 from left to right.
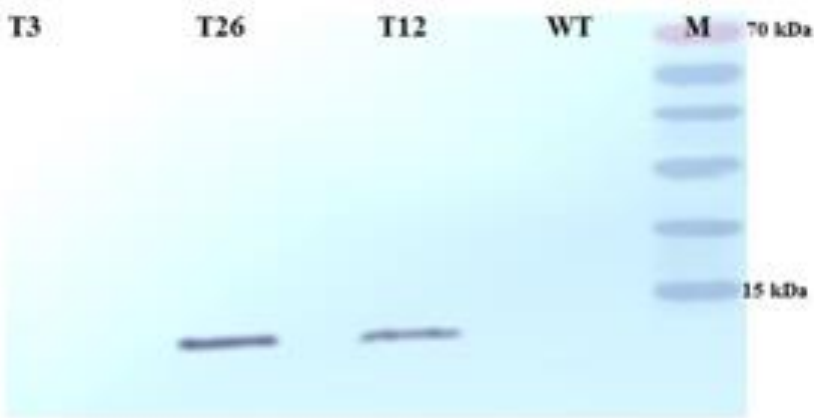

Figure 3. Western-blot analysis of transgenic plants. T3: Protein sample of transgenic line T3, WT: Protein sample of wild type plant, T26: Protein sample of transgenic line T26, T12: Protein sample of transgenic line T12

\subsection{Induction of Immune Response in Immunized}

Rabbit

Rabbits were intraperitoneally immunized on days 0,15 , 29 , and 50 with $500 \mu \mathrm{g}$ of the crude protein from the wild

type and the transgenic plant (T26). Extract of nontransgenic plants was used in the control group. Animals 
were then bled and the sera was analyzed for the presence of anti-NDV antibodies in the ELISA assay. Antibodies produced in the experimentally-immunized Rabbit -i.e, Rabbit receiving transformed plant extracts- showed a strong response against NDV when tested in ELISA. No response was detected in the rabbits immunized with non-transformed plants (Fig. 4).

1.2

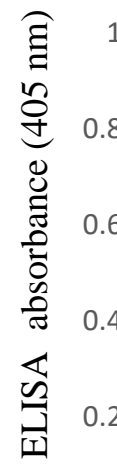

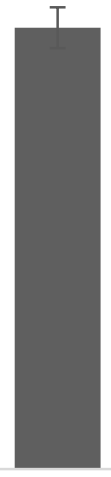

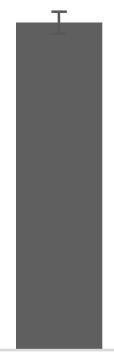

2

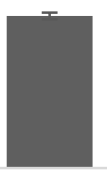

3

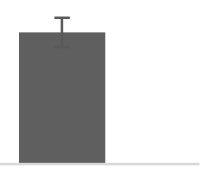

4

Figure 4. Induction of specific antibody in serum sample of rabbits injected with the transgenic plant extract; 1: The positive control (commercial vaccine), 2: The serum of rabbit immunized by injection of transgenic plant extract; 3: The serum of rabbit immunized by injection of Nontransformed Plants, 4: BSA

\section{Discussion}

Production of therapeutic proteins including recombinant vaccines in plants represents an economical alternative to both traditional inactivation of infectious agents and the fermentation-based expression systems, especially for the production of high-volume reserves of subunit vaccines. Since the early 1990's, there have been numerous attempts to produce recombinant vaccines against various human and animal diseases in transgenic plant and various plant species have been investigated as the host for production of recombinant vaccines (1). Oral delivery of antigens expressed in plant parts is possible because the plant cell wall provides an effective protection against degradation; consequently, much of the vaccine antigen expressed in the cells can reach the gutassociated lymphoid tissue (GALT) in an immunogenic form (8). The property of plant cell walls in protecting the antigens from gastric digestion makes the plant systems ideal platforms for the production of recombinant vaccines (1).

Although the traditional inactivated vaccines against ND are effective in protecting the animal, there are major disadvantages in using this vaccine that makes the development of alternative methods necessary (9). In recent years, there have been extended efforts to develop new subunit vaccines against $\mathrm{ND}$ and other avian diseases $(6,7,10,11)$. Ge et al. (2013), for example, reported development of a chimeric recombinant vaccine that protects against Newcastle disease, as well as infectious bursal disease (9).
Particularly, Hemagglutinin-Neuraminidase (HN) and Fusion (F) epitopes have been of interest for the development of anti-ND recombinant vaccines. For example, Lai et al. (2013) expressed hemagglutininneuraminidase protein in transgenic tobacco cells and used the recombinant protein for immunization of mice. The authors reported induction of specific anti-NDV antibodies in the mice receiving the recombinant protein (12). Similarly, Yang et al. (2016) observed that recombinant infectious bronchitis virus expressing the hemagglutinin-neuraminidase $(\mathrm{HN})$ protects chickens against NDV challenge (13). Shahriari et al. (2015) expressed fusion (F) and haemagglutininneuraminidase (HN) epitopes in hairy roots of tobacco (Nicotiana tabaccum) to develop a scalable platform for production of anti-ND recombinant vaccines (14). Induction of immune response in animal host is the final step in every project concerning the development of recombinant vaccine. An antigen expressed in transgenic plant can be considered a potential recombinant vaccine only if its ability to induce immune response is approved through animal model trials (1). In a pioneering work, Dalsgaard et al. (1997) demonstrated that subcutaneous injection of the recombinant VP2 vaccine expressed in black-eyed bean conferred protection against clinical disease in mink (15). Alvarez et al. (2006) reported that plant-made subunit vaccine against plague was able to induce immune response in mice when administered orally (8). Similarly, Chishester et al. (2012) reported that the recombinant Hemagglutinin-based influenza vaccine 
produced in transgenic plant induced strong immune response in animal model (16).

In the present research, subcutaneous administration of the recombinant HN-F protein induced production of specific anti-ND antibodies in rabbits. This finding provides further support for the notion that plant-based recombinant vaccines are effective in induction of immune response in animal hosts. Our results match those reported by other authors. For example, Yang et al. (2007) expressed fusion glycoprotein of Newcastle disease virus in transgenic rice and observed that the recombinant protein is able to induce immune response in mice (17). Berinstein et al. (2005) reported induction of both mucosal and systemic immunity in animal hosts as treated with recombinant protein expressed in potato (18). Hahn et al. (2007) expressed hemagglutininneuraminidase protein of Newcastle disease virus in transgenic tobacco. The authors fed chickens with recombinant protein and observed induction of specific antibodies in the treated chickens (19).

\section{Conclusions}

The results obtained in this research indicated that recombinant vaccines generated in plant host possess high potential for the development of new generation of vaccines against Newcastle disease. We believe that our results provide additional support for the feasibility of using transgenic plants as an effective system for the production of recombinant vaccines. Application of other immunogenic sites of NDV capsid protein along with other kinds of promoters, enhancers, ribosome binding sites, signal peptides, and any molecular element involving in enhancement of foreign gene expression could be the subject of future studies.

\section{References}

1. Habibi-Pirkoohi M, Mohkami A. Recombinant vaccine production in green plants: State of art. J Cell Molecul Res 2015;7(1):59-67.

2. Zhang L, Zhang J, Chen HT, Zhou JH, Ma LN, Ding $\mathrm{YZ}$, et al. Research in advance for FMD novel vaccines. Virol J. 2011;8(1):268. doi: 10.1186/1743-422X-8-268 pmid: 21635788

3. Sala F, Manuela Rigano M, Barbante A, Basso B, Walmsley AM, Castiglione S. Vaccine antigen production in transgenic plants: strategies, gene constructs and perspectives. Vaccine. 2003;21(78):803-808. doi: $10.1016 / \mathrm{s} 0264-410 x(02) 00603-5$ pmid: 12531364

4. Habibi-Pirkoohi M, Malekzadeh-Shafaroudi S, Marashi H, Moshtaghi N, Nassiri M, Zibaee S. Transient Expression of Foot and Mouth Disease Virus (FMDV)Coat Protein in Tobacco (Nicotiana tabacom) via Agroinfiltration. Iran J Biotechnol 2014;12(3):28-35. doi: 10.15171/ijb.1015

5. Kong Q, Richter L, Yang YF, Arntzen CJ, Mason HS, Thanavala Y. Oral immunization with hepatitis B surface antigen expressed in transgenic plants. Proc Natl Acad Sci U S A. 2001;98(20):11539-11544. doi: 10.1073/pnas. 191617598 pmid: 11553782
6. Nagy A, Lee J, Mena I, Henningson J, Li Y, Ma J, et al. Recombinant Newcastle disease virus expressing H9 HA protects chickens against heterologous avian influenza H9N2 virus challenge. Vaccine. 2016;34(23):2537-2545. doi: 10.1016/j.vaccine.2016. 04.022 pmid: 27102817

7. Shahriari AG, Bagheri A, Bassami MR, MalekzadehShafaroudi S, Afsharifar A, Niazi A. Expression of Hemagglutinin-Neuraminidase and fusion epitopes of Newcastle Disease Virus in transgenic tobacco. Electr J Biotechnol 2016;22:38-43. doi: 10.1016/j.ejbt.2016.05. 003

8. Alvarez ML, Pinyerd HL, Crisantes JD, Rigano MM, Pinkhasov J, Walmsley AM, et al. Plant-made subunit vaccine against pneumonic and bubonic plague is orally immunogenic in mice. Vaccine. 2006;24(14):24772490. doi: 10.1016/j.vaccine.2005.12.057 pmid: 16442 673

9. Ge J, Wang X, Tian M, Wen Z, Feng Q, Qi X, et al. Novel in-ovo chimeric recombinant Newcastle disease vaccine protects against both Newcastle disease and infectious bursal disease. Vaccine. 2014;32(13):1514-1521. doi: 10.1016/j.vaccine.2014.01.020 pmid: 24486349

10. Ngu LN, Nji NN, Ambada G, Ngoh AA, Njambe Priso GD, Tchadji JC, et al. Dendritic cell targeted HIV-1 gag protein vaccine provides help to a recombinant Newcastle disease virus vectored vaccine including mobilization of protective CD8(+) $\mathrm{T}$ cells. Immun Inflamm Dis. 2018;6(1):163-175. doi: 10.1002/iid3 .209 pmid: 29205929

11. Morgan RW, Gelb J, Jr., Schreurs CS, Lutticken D, Rosenberger JK, Sondermeijer PJ. Protection of chickens from Newcastle and Marek's diseases with a recombinant herpesvirus of turkeys vaccine expressing the Newcastle disease virus fusion protein. Avian Dis. 1992;36(4):858-870. doi: 10.2307/1591544 pmid: 1485872

12. Lai KS, Yusoff K, Mahmood M. Functional ectodomain of the hemagglutinin-neuraminidase protein is expressed in transgenic tobacco cells as a candidate vaccine against Newcastle disease virus. Plant Cell Tissue Organ Cult. 2012;112(1):117-121. doi: 10.1007/s112 40-012-0214-X

13. Yang $\mathrm{X}$, Zhou $\mathrm{Y}, \mathrm{Li}$ J, Fu L, Ji G, Zeng F, et al. Recombinant infectious bronchitis virus (IBV) H120 vaccine strain expressing the hemagglutininneuraminidase $(\mathrm{HN})$ protein of Newcastle disease virus (NDV) protects chickens against IBV and NDV challenge. Arch Virol. 2016;161(5):1209-1216. doi: 10.1007/s00705-016-2764-4 pmid: 26873815

14. Shahriari AG, Bagheri A, Bassami MR, Malekzadeh Shafaroudi S, Afsharifar AR. Cloning and expression of fusion $(\mathrm{F})$ and haemagglutinin-neuraminidase $(\mathrm{HN})$ epitopes in hairy roots of tobacco (Nicotiana tabaccum) as a step toward developing a candidate recombinant vaccine against Newcastle disease. J Cell Molecul Res 2015;7(1):11-18.

15. Dalsgaard $\mathrm{K}$, Uttenthal $\mathrm{A}$, Jones $\mathrm{TD}, \mathrm{Xu} \mathrm{F}$, Merryweather A, Hamilton WD, et al. Plant-derived vaccine protects target animals against a viral disease. Nat Biotechnol. 1997;15(3):248-252. doi: 10.1038/nb t0397-248 pmid: 9062924

16. Chichester JA, Jones RM, Green BJ, Stow M, Miao F, Moonsammy G, et al. Safety and immunogenicity of a 
plant-produced recombinant hemagglutinin-based influenza vaccine (HAI-05) derived from A/Indonesia/05/2005 (H5N1) influenza virus: a phase 1 randomized, double-blind, placebo-controlled, doseescalation study in healthy adults. Viruses. 2012;4(11):3227-3244. doi: 10.3390/v4113227 pmid: 23202523

17. Yang ZQ, Liu QQ, Pan ZM, Yu HX, Jiao XA. Expression of the fusion glycoprotein of Newcastle disease virus in transgenic rice and its immunogenicity in mice. Vaccine. 2007;25 (4):591-598. doi: 10.1016/j.vaccine.2006.08.0 16 pmid: 17049688
18. Berinstein A, Vazquez-Rovere C, Asurmendi S, Gomez $\mathrm{E}$, Zanetti F, Zabal O, et al. Mucosal and systemic immunization elicited by Newcastle disease virus (NDV) transgenic plants as antigens. Vaccine. 2005;23(48-49):5583-5589. doi: 10.1016/j.vaccine.2 005.06.033 pmid: 16099555

19. Hahn B-S, Jeon I-S, Jung Y-J, Kim J-B, Park J-S, Ha S-H, et al. Expression of hemagglutinin-neuraminidase protein of Newcastle disease virus in transgenic tobacco. Plant Biotechnol Rep 2007;1(2):85-92. doi: 10.1007/s11816-007-0012-9 